\title{
Erratum to: Structure of the solution set to impulsive functional differential inclusions on the half-line
}

\author{
Grzegorz Gabor and Agata Grudzka
}
Erratum to: Nonlinear Differ. Equ. Appl. 19 (2012), 609-627 DOI 10.1007/s00030-011-0144-z

\begin{abstract}
The aim of the paper is twofold. At first, it is an erratum to: Gabor G., Grudzka A.: Structure of the solution set to impulsive functional differential inclusions on the half-line. NoDEA 19, 609-627 (2012). We fill a little gap in a proof of Theorem 3.5 by a change of a compactness type condition, and give a discussion on assumptions sufficient to obtain an $R_{\delta}$-structure of solution sets. Simultaneously, we improve the main theorems by the use of modified proof-arguments. Namely, the assumptions on impulse maps can be weakened.
\end{abstract}

\section{Introduction}

In the original paper a structure of the solution set of the following impulsive Cauchy problem governed by a semilinear differential inclusion on a noncompact interval has been studied:

$$
\begin{cases}\dot{y}(t) \in A(t) y(t)+F\left(t, y_{t}\right), & \text { for a.a. } t \in[0, \infty), t \neq t_{k}, k \in \mathbb{N} \\ y(t)=x(t), & \text { for } t \in[-\tau, 0] \\ y\left(t_{k}^{+}\right)=y\left(t_{k}\right)+I_{k}\left(y_{t_{k}}\right), & \text { for } k \in \mathbb{N}^{+}\end{cases}
$$

where $\{A(t)\}_{t \in[0, \infty)}$ is a family of linear operators in a Banach space $E$ generating an evolution operator; $F$ is an upper-Carathéodory map; $\tau>0$ is a given number, $y_{t}(\theta)=y(t+\theta), \theta \in[-\tau, 0] ; I_{k}$ are impulse functions, $k \in \mathbb{N}$,

The online version of the original article can be found under doi:10.1007/s00030-011-0144-z. This research was partially supported by the Polish NCN Grant No. 2013/09/B/ST1/01963. 
$y\left(t^{+}\right)=\lim _{s \rightarrow t^{+}} y(s)$, and the time sequence $\left(t_{k}\right)_{k \in \mathbb{N}}$ is an increasing sequence of given points in $[0, \infty)$ without accumulation points. The history of research in this topic is described in the original paper. As an intermediate step in considerations the following problem on a compact interval has been investigated

$$
\begin{cases}\dot{y}(t) \in A(t) y(t)+F\left(t, y_{t}\right), & \text { for a.e. } t \in\left[0, t_{m}\right], t \neq t_{k}, k<m, \\ y(t)=x(t), & \text { for } t \in[-\tau, 0] \\ y\left(t_{k}^{+}\right)=y\left(t_{k}\right)+I_{k}\left(y_{t_{k}}\right), & \text { for } k<m .\end{cases}
$$

One of the main results of the original paper reads as follows:

Theorem 1.1. (Theorem 3.5 in the original paper) Let $E$ be a Banach space and let hypothesis

$(A)_{m}\{A(t)\}_{t \in\left[0, t_{m}\right]}$ is a family of linear (not necessarily bounded) operators $\left(A(t): D(A) \subset E \rightarrow E, t \in\left[0, t_{m}\right], D(A)\right.$ a dense subset of $E$ not depending on $t)$ generating an evolution operator $T: \Delta_{m} \rightarrow \mathcal{L}(E)$,

hold, where $\Delta_{m}=\left\{(t, s) \in\left[0, t_{m}\right] \times\left[0, t_{m}\right] \mid s \leq t \leq t_{m}\right\}$. Suppose that the multivalued map $F:\left[0, t_{m}\right] \times \mathcal{C}([-\tau, 0], E) \rightarrow P_{c p, c v}(E)$, where $P_{c p, c v}(E)$ is a family of nonempty compact convex subsets of $E$, satisfies conditions

$(F 1)_{m} F(\cdot, c)$ has a strongly measurable selection for every $c \in \mathcal{C}([-\tau, 0], E)$, $(F 2)_{m} F(t, \cdot)$ is u.s.c. for a.e. $t \in\left[0, t_{m}\right]$,

$(F 3)_{m} F$ for almost every $t \in\left[0, t_{m}\right]$ has at most a linear growth, i.e., there exists a function $\alpha \in L^{1}\left(\left[0, t_{m}\right]\right)$ such that

$$
\|F(t, c)\| \leq \alpha(t)\left(1+\|c\|_{\mathcal{C}}\right) \quad \text { for a.e. } t \in\left[0, t_{m}\right],
$$

$(F 4)_{m}$ There exists a function $\mu \in L^{1}\left(\left[0, t_{m}\right]\right)$ such that

$$
\beta(F(t, D)) \leq \mu(t) \sup _{-\tau \leq \theta \leq 0} \beta(D(\theta))
$$

for a.e. $t \in\left[0, t_{m}\right]$ and for every bounded $D \subset \mathcal{C}([-\tau, 0], E)$, where $D(\theta)=\{c(\theta) \mid c \in D\}$.

Moreover, assume that the maps $I_{k}: \mathcal{C}([-\tau, 0], E) \rightarrow E, k \in \mathbb{N}$, are continuous and there exist constants $r_{k}>0$ such that

$$
\beta\left(I_{k}(D)\right) \leq r_{k} \sup _{-\tau \leq \theta \leq 0} \beta(D(\theta))
$$

for every bounded $D \subset \mathcal{C}([-\tau, 0], E)$,

$$
\sum_{k=1}^{m} r_{k}<\frac{1}{B_{m}}
$$

where $B_{m}=\sup _{(t, s) \in \Delta_{m}}\|T(t, s)\|_{\mathcal{L}(E)}$.

Then the solution set for problem $(2)$ is an $R_{\delta}$-set in $P C\left(\left[0, t_{m}\right], E\right)[x]$. 
Recall that $\mathcal{C}([-\tau, 0], E)$ is the space of piecewise continuous functions $c:[-\tau, 0] \rightarrow E$ with finite number of discontinuity points $\left\{t_{*}\right\}$ such that $t_{*} \neq 0$ and all values

$$
c\left(t_{*}^{+}\right)=\lim _{h \rightarrow 0^{+}} c\left(t_{*}+h\right) \text { and } c\left(t_{*}^{-}\right)=\lim _{h \rightarrow 0^{-}} c\left(t_{*}+h\right)
$$

are finite. We consider in $\mathcal{C}([-\tau, 0], E)$ the $L^{1}$-norm $\|c\|_{\mathcal{C}}=\int_{-\tau}^{0}\|c(t)\| d t$. We also denote

$$
P C\left(\left[0, t_{m}\right], E\right)[x]:=\left\{y[x] \in P C\left(\left[-\tau, t_{m}\right], E\right) \mid y \in P C\left(\left[0, t_{m}\right], E\right)\right\},
$$

where $y[x]$ is equal $y$ on $\left[0, t_{m}\right]$ and $y[x]=x$ on $[-\tau, 0]$.

In Step 3 of the proof of Theorem 1.1 (see the original paper) one has applied assumption $(F 4)_{m}$ to estimate a measure of noncompactness of some sequence of functions. Unfortunately, it has been done incorrectly, because the inequality

$$
\beta\left[F\left(\{s\} \times B\left(\left\{y_{n_{s}}\right\}_{n \geq p}, 3 d_{p}\right)\right)\right] \leq \mu(s) \cdot \sup _{-\tau \leq \theta \leq 0} \beta\left(B\left(\left\{y_{n}(s+\theta)\right\}_{n \geq p}, 3 d_{p}\right)\right)
$$

could be false in the $L^{1}$ norm in $\mathcal{C}([-\tau, 0], E)$. Below, in Sect. 2, we give an assumption which is sufficient for the proof, and discuss differences between the assumptions. In fact we really hope that assumption $(F 4)_{m}$ is also sufficient but we have to leave this hypothesis as an open question.

In Sect. 3 we give a corrected proof, but also improve the results and show that assumption (I2) is not needed. To prove this we will use in a proof different arguments from those in the original paper.

\section{Compactness type assumptions on $F$}

Instead of $(F 4)_{m}$ we assume

$(F 4)_{m}^{\prime}$ for every $\varepsilon>0$ and every bounded set $D \subset \mathcal{C}([-\tau, 0], E)$ there exist $\delta>0$ and a function $\mu \in L^{1}\left(\left[0, t_{m}\right]\right)$ such that

$$
\beta\left(F\left(t, O_{\delta}(D)\right)\right) \leq \mu(t) \sup _{-\tau \leq \theta \leq 0} \beta\left(O_{\varepsilon}(D(\theta))\right) \quad \text { for a.e. } t \in\left[0, t_{m}\right],
$$

where $O_{\delta}(D)$ denotes a $\delta$-neighborhood of $D$ defined as $O_{\delta}(D):=\{z \in$ $\mathcal{C}([-\tau, 0], E) ; \operatorname{dist}(z, D)<\delta\}$.

It is easy to see that $(F 4)_{m}^{\prime} \Longrightarrow(F 4)_{m}$. Indeed, for every bounded set $D \subset \mathcal{C}([-\tau, 0], E)$ and every $\varepsilon>0$ we take $\delta>0$ and $\mu \in L^{1}\left(\left[0, t_{m}\right]\right)$ from $(F 4)_{m}^{\prime}$, and obtain, for a.e., $t \in\left[0, t_{m}\right]$,

$$
\beta(F(t, D)) \leq \beta\left(F\left(t, O_{\delta}(D)\right)\right) \leq \mu(t) \sup _{-\tau \leq \theta \leq 0} \beta\left(O_{\varepsilon}(D(\theta))\right) .
$$

Since $\varepsilon$ was arbitrary, we get $(F 4)_{m}$.

Assumption $(F 4)_{m}^{\prime}$ is satisfied by many important classes of maps. We list some of them in the following three examples.

Example 2.1. Each completely continuous map with respect to the second variable satisfies $(F 4)_{m}^{\prime}$ with arbitrary $\delta>0$ and $\mu \in L^{1}\left(\left[0, t_{m}\right]\right)$. 
Example 2.2. Let $F(t, y)=\alpha(t) \int_{-\tau}^{0} y(\theta) d \theta$. As we can see, this perturbation $F$ depends on the behavior of $y$ during the whole time interval $[-\tau, 0]$. Notice that, for any bounded set $D$, we get

$$
\begin{aligned}
\beta\left(F\left(t, O_{\delta}(D)\right)\right) & =\alpha(t) \beta\left(\left\{\int_{-\tau}^{0} z(\theta) d \theta \mid \operatorname{dist}(z, D) \leq \delta\right\}\right) \\
& \leq \alpha(t)\left(\beta\left(\left\{\int_{-\tau}^{0} y(\theta) d \theta \mid y \in D\right\}\right)+\delta\right)
\end{aligned}
$$

If $\sup _{-\tau \leq \theta \leq 0} \beta(\{y(\theta) \mid y \in D\})=+\infty$, then condition $(F 4)_{m}^{\prime}$ is obviously fulfilled. If $\sup _{-\tau \leq \theta \leq 0} \beta(\{y(\theta) \mid y \in D\})<+\infty$, then the constant function $[-\tau, 0] \ni s \mapsto \sup _{-\tau \leq \theta \leq 0} \beta(\{y(\theta) \mid y \in D\})$, is integrable. Moreover, $\{y(\theta) \mid y \in$ $D\} \subset B(0, r)$ for some $r>0$ and every $\theta \in[-\tau, 0]$. Therefore the family $D$ is uniformly integrably bounded. Hence,

$$
\begin{aligned}
\beta\left(\left\{\int_{-\tau}^{0} y(\theta) d \theta \mid y \in D\right\}\right) & \leq 4 \int_{-\tau}^{0} \sup _{-\tau \leq \theta \leq 0} \beta(\{y(\theta) \mid y \in D\}) d \theta \\
& =4 \tau \sup _{-\tau \leq \theta \leq 0} \beta(\{y(\theta) \mid y \in D\}),
\end{aligned}
$$

and, consequently,

$$
\begin{aligned}
& \beta\left(F\left(t, O_{\delta}(D)\right)\right) \leq \alpha(t)\left[4 \tau \sup _{-\tau \leq \theta \leq 0} \beta(\{y(\theta) \mid y \in D\})+\delta\right] \\
& =4 \tau \alpha(t)\left[\sup _{-\tau \leq \theta \leq 0} \beta(\{y(\theta) \mid y \in D\})+\frac{\delta}{4 \tau}\right] .
\end{aligned}
$$

It is sufficient to take $\mu(t):=4 \tau \alpha(t)$ and $\delta:=4 \tau \varepsilon$.

Example 2.3. Assume that $F$ satisfies $(F 4)_{m}$ and a Lipschitz condition with respect to the second variable, i.e., there exists a function $L \in L^{1}\left(\left[0, t_{m}\right]\right)$ such that for every $t \in\left[0, t_{m}\right]$ and each $y, z \in \mathcal{C}([-\tau, 0], E)$ one has

$$
d_{H}(F(t, z), F(t, y)) \leq L(t)\|z-y\|_{\mathcal{C}} .
$$

Then $F$ satisfies $(F 4)_{m}^{\prime}$.

Indeed, by the Lipschitz condition we have

$$
F\left(s, O_{\delta}(D)\right) \subset O_{L(s) \delta}(F(s, D))
$$

for every $s \in\left[0, t_{m}\right]$ and $\delta>0$. Thus

$$
\begin{aligned}
\beta\left(F\left(\{s\} \times O_{\delta}(D)\right)\right) & \leq \beta(F(s, D))+L(s) \delta \\
& \leq \mu(s) \sup _{-\tau \leq \theta \leq 0} \beta(D(\theta))+L(s) \delta \\
& \leq \tilde{\mu}(s)\left(\sup _{-\tau \leq \theta \leq 0} \beta(D(\theta))+\delta\right),
\end{aligned}
$$

where $\tilde{\mu}(s):=\max \{\mu(s), L(s)\}$. It is sufficient to take $\delta=\varepsilon$. 


\section{Main results}

The main goal of this paper is to prove the following result on a topological structure of the solution set for problem (2), and, as a consequence, an analogous result for the problem on a noncompact interval.

Theorem 3.1. Let $E$ be a Banach space, let hypotheses $(A)_{m},(F 1)_{m}-(F 3)_{m}$, $(F 4)_{m}^{\prime}$ hold and let the maps $I_{k}: \mathcal{C}([-\tau, 0], E) \rightarrow E, k \in \mathbb{N}$, be continuous and satisfy (I1).

Then the solution set for problem $(2)$ is an $R_{\delta}$-set in $P C\left(\left[0, t_{m}\right], E\right)[x]$.

Proof. We follow the steps of the proof of Theorem 3.5 in the original paper, and make suitable changes.

Step 1. We show (see the original paper) that all solutions to (2) are pointwise equibounded by some constant $\bar{K}_{m}$. Let $\tilde{K}_{m}=\tau \bar{K}_{m}$. Then $\left\|y_{t}\right\|_{\mathcal{C}} \leq \tilde{K}_{m}$ for every solution $y$ to $(2)$. We define a mapping $\tilde{F}:\left[0, t_{m}\right] \times \mathcal{C}([-\tau, 0], E) \rightarrow$ $P_{c p, c v}(E)$,

$$
\tilde{F}(t, c)= \begin{cases}F(t, c), & \text { if } t \in\left[0, t_{m}\right] \text { and }\|c\|_{\mathcal{C}} \leq \tilde{K}_{m}, \\ F\left(t, \frac{\tilde{K}_{m c}}{\|c\|_{\mathcal{c}}}\right), & \text { if } t \in\left[0, t_{m}\right] \text { and }\|c\|_{\mathcal{C}}>\tilde{K}_{m}\end{cases}
$$

which is integrably bounded and generates the same set of solutions, if we replace $F$ by $\tilde{F}$ in (2). In conclusion, without any loss of generality we can assume

$\left(F 3^{\prime}\right)_{m}\|F(t, c)\| \leq \psi_{m}(t)$ for every $t \in\left[0, t_{m}\right]$, where $\psi_{m} \in L^{1}\left(\left[0, t_{m}\right]\right)$ instead of $(F 3)_{m}$.

Step 2. Repeating the lines of Step 2 in the original paper we construct a sequence of multivalued maps $\left\{G_{n}\right\}_{n=1}^{\infty}, G_{n}:\left[0, t_{m}\right] \times \mathcal{C}([-\tau, 0], E) \rightarrow P_{c l, c v}(E)$ such that:

(i) each multivalued map $G_{n}(t, \cdot): \mathcal{C}([-\tau, 0], E) \rightarrow P_{c l, c v}(E), n \geq 1$, is continuous for a.e. $t \in\left[0, t_{m}\right]$,

(ii) $F(t, c) \subset \cdots \subset G_{n+1}(t, c) \subset G_{n}(t, c) \subset \overline{\operatorname{conv}} F\left(t, B_{3 d_{n}}(c)\right)$, where $d_{n}=$ $\frac{1}{3^{n}}, n \geq 1$

(iii) $F(t, c)=\bigcap_{n>1} G_{n}(t, c)$,

(iv) for each $n \geq 1$ there exists a selection $g_{n}:\left[0, t_{m}\right] \times \mathcal{C}([-\tau, 0], E) \rightarrow E$ of $G_{n}$, such that $g_{n}(\cdot, c)$ is measurable and $g_{n}(t, \cdot)$ is locally Lipschitz.

Step 3. We consider the differential problem:

$$
\begin{cases}\dot{y}(t) \in A(t) y(t)+G_{n}\left(t, y_{t}\right), & \text { for a.e. } t \in\left[0, t_{m}\right], t \neq t_{k}, k<m, \\ y(t)=x(t), & \text { for } t \in[-\tau, 0], \\ y\left(t_{k}^{+}\right)=y\left(t_{k}\right)+I_{k}\left(y_{t_{k}}\right), & \text { for } k<m,\end{cases}
$$

and denote by $S_{m}^{n}$ the solution set of problem (4). From condition (ii) it follows that there is a constant $K_{m} \geq 0$ such that $\|y\| \leq K_{m}$ for every $y \in S_{m}^{n}$.

We show that each sequence $\left\{y_{n}\right\}$ such that $y_{n} \in S_{m}^{n}$ for all $n \geq 1$ has a convergent subsequence $y_{n_{k}} \rightarrow y \in S_{m}$. Notice that $y_{n}(t)=x(t)$ for every $t \in[-\tau, 0]$. 
Consider the sequence $\left\{y_{n}\right\}$ on $\left[0, t_{1}\right]$. Then

$$
y_{n}(t)=T(t, 0) x(0)+\int_{0}^{t} T(t, s) f_{n}(s) d s
$$

for $t \in\left[0, t_{1}\right]$, where $f_{n} \in L^{1}\left(\left[0, t_{1}\right], E\right), f_{n}(t) \in G_{n}\left(t,\left(y_{n}\right)_{t}\right)$ for a.e. $t \in\left[0, t_{1}\right]$.

For $d_{k}, k \geq 1$, from $(F 4)_{m}^{\prime}$ we get $\delta>0$ such that

$$
\beta\left(F\left(\{s\} \times O_{\delta}\left(\left\{\left(y_{n}\right)_{s}\right\}_{n \geq 1}\right)\right)\right) \leq \mu(s)\left[\sup _{-\tau \leq \theta \leq 0} \beta\left(\left\{\left(y_{n}\right)_{s}(\theta)\right\}_{n \geq 1}\right)+d_{k}\right] .
$$

Take $d_{l}, l \geq k$, such that $3 d_{l}<\delta$. Then

$$
\begin{aligned}
\beta\left(\left\{f_{n}(s)\right\}_{n \geq 1}\right) & =\beta\left(\left\{f_{n}(s)\right\}_{n \geq l}\right) \leq \beta\left[F\left(\{s\} \times O_{3 d_{l}}\left(\left\{\left(y_{n}\right)_{s}\right\}_{n \geq l}\right)\right)\right] \\
& \leq \mu(s)\left[\sup _{-\tau \leq \theta \leq 0} \beta\left(\left\{y_{n}(s+\theta)\right\}_{n \geq l}\right)+d_{k}\right] \\
& \leq \mu(s)\left[\max \left(\sup _{-\tau \leq \sigma \leq 0} \beta(\{x(\sigma)\}), \sup _{0 \leq \sigma \leq s} \beta\left(\left\{y_{n}(\sigma)\right\}_{n \geq l}\right)\right)+d_{k}\right] \\
& =\mu(s)\left(\bar{\rho}(s)+d_{k}\right),
\end{aligned}
$$

where $\bar{\rho}(s):=\sup _{0 \leq \sigma \leq s} \beta\left(\left\{y_{n}(\sigma)\right\}_{n \geq 1}\right)$. This implies that

$$
\begin{aligned}
\beta\left(\left\{y_{n}(t)\right\}_{n \geq 1}\right) & =\beta\left(\left\{T(t, 0) x(0)+\int_{0}^{t} T(t, s) f_{n}(s) d s\right\}_{n \geq 1}\right) \\
& \leq 2 B_{m} \int_{0}^{t} \mu(s)\left(\bar{\rho}(s)+d_{k}\right) d s
\end{aligned}
$$

for every $k \geq 1$. But $d_{k} \searrow 0$ when $k \rightarrow \infty$, so

$$
\bar{\rho}(t) \leq 2 B_{m} \int_{0}^{t} \mu(s) \bar{\rho}(s) d s .
$$

From the Gronwall inequality we obtain $\bar{\rho}(t)=0$, which means that, in the interval $\left[0, t_{1}\right]$,

$$
\beta\left(\left\{y_{n}(t)\right\}_{n \geq 1}\right)=0 .
$$

Hence, $\beta\left(\left\{f_{n}(s)\right\}_{n \geq 1}\right)=0$ for $s \leq t_{1}$.

For $t=t_{1}$ one has

$$
\begin{aligned}
\beta & \left(\left\{T\left(t, t_{1}\right) I_{1}\left(\left(y_{n}\right)_{t_{1}}\right)\right\}_{n \geq 1}\right)=\beta\left(\left\{T\left(t, t_{1}\right) I_{1}\left(\left(y_{n}\right)_{t_{1}}\right)\right\}_{n \geq l}\right) \\
& \leq\left\|T\left(t, t_{1}\right)\right\| \cdot \beta\left(\left\{I_{1}\left(\left(y_{n}\right)_{t_{1}}\right)\right\}_{n \geq l}\right) \\
& \left.\leq B_{m} p_{1} \sup _{-\tau \leq \theta \leq 0} \beta\left(\left\{\left(y_{n}\right)\right)_{t_{1}}(\theta)\right\}_{n \geq l}\right) \\
& \leq B_{m} p_{1} \cdot \max \left(\sup _{-\tau \leq \sigma \leq 0} \beta(\{x(\sigma)\}), \sup _{0 \leq \sigma \leq t_{1}} \beta\left(\left\{y_{n}(\sigma)\right\}_{n \geq 1}\right)\right) \\
& =B_{m} p_{1} \bar{\rho}\left(t_{1}\right)=0 .
\end{aligned}
$$

Every solution $y_{n}$ has the form

$$
y_{n}(t)=T\left(t, t_{1}\right) y_{n}\left(t_{1}\right)+T\left(t, t_{1}\right) I_{1}\left(\left(y_{n}\right)_{t_{1}}\right)+\int_{t_{1}}^{t} T(t, s) f_{n}(s) d s,
$$


for $t \in\left[t_{1}, t_{2}\right]$, where $f_{n} \in L^{1}\left(\left[t_{1}, t_{2}\right], E\right), f_{n}(t) \in G_{n}\left(t,\left(y_{n}\right)_{t}\right)$ for a.e. $t \in$ $\left[t_{1}, t_{2}\right]$. Analogously as before one shows that $\beta\left(\left\{f_{n}(s)\right\}_{n>1}\right)=0$. Next, we consider $t=t_{2}$ and so on. Finally, $\beta\left(\left\{y_{n}(t)\right\}_{n \geq 1}\right)=0$ for $t \in\left[0, t_{m}\right]$, and hence $\beta\left(\left\{f_{n}(t)\right\}_{n \geq 1}\right)=0$.

For $t \leq t^{\prime}$ in $\left[0, t_{1}\right]$ we have

$$
\left\|y_{n}\left(t^{\prime}\right)-y_{n}(t)\right\|=\left\|\int_{t}^{t^{\prime}} \dot{y}_{n}(s) d s\right\| \leq B_{m} \int_{t}^{t^{\prime}} \psi_{m}(s) d s,
$$

since $\left\|G_{n}(t, c)\right\| \leq \psi_{m}(t)$ for a.e. $t \in\left[0, t_{m}\right]$ from (ii) and $(F 3)_{m}^{\prime}$. Therefore, the family $\left\{y_{n}\right\}$ is equicontinuous. This implies the existence of a convergent subsequence on $\left[0, t_{1}\right]$ denoted by $\left(y_{n_{q_{1}}}^{1}\right)_{q_{1} \in Q_{1}}$. Define

$$
y_{n_{q_{1}, 1}}(t):= \begin{cases}y_{n_{q_{1}}}^{1}(t), & \text { for } t \in\left[0, t_{1}\right], \\ y_{n_{q_{1}}}^{1}\left(t_{1}\right)+I_{1}\left(\left(y_{n_{q_{1}}}^{1}\right)_{t_{1}}\right) & \\ \quad+\sum_{t_{1}<t_{j}<t} T\left(t, t_{j}\right) I_{j}\left(\left(y_{n_{q_{1}}}\right)_{t_{j}}\right) & \\ \quad+\int_{t_{1}}^{t} T(t, s) f_{n_{q_{1}}}(s) d s, & \text { for } t>t_{1} .\end{cases}
$$

Let $y_{n_{q_{1}}}^{2}:=\left.y_{n_{q_{1}, 1}}\right|_{\left[t_{1}, t_{2}\right]}$. Notice that $y_{n_{q_{1}}}^{2}\left(t_{1}^{+}\right)=y_{n_{q_{1}}}^{1}\left(t_{1}^{+}\right)$for every $k \geq 1$. For $t \leq t^{\prime} \mathrm{w}\left[t_{1}, t_{2}\right]$ we have

$$
\left\|y_{n_{q_{1}}}^{2}\left(t^{\prime}\right)-y_{n_{q_{1}}}^{2}(t)\right\| \leq B_{m} \int_{t}^{t^{\prime}} \psi_{m}(s) d s
$$

so the family $\left\{y_{n_{q_{1}}}^{2}\right\}$ is equicontinuous. Thus it contains a convergent subsequence $\left(y_{n_{q_{2}}}^{2}\right)_{q_{2} \in Q_{2}}$ on $\left[t_{1}, t_{2}\right]$, where $Q_{2} \subset Q_{1}$. We take a concatenation

$$
y_{n_{q_{2}, 2}}(t):= \begin{cases}y_{n_{q_{2}}}^{1}(t), & \text { dla } t \in\left[0, t_{1}\right], \\ y_{n_{q_{2}}}^{2}(t), & \text { dla } t \in\left(t_{1}, t_{2}\right], \\ y_{n_{q_{2}}}^{2}\left(t_{2}\right)+I_{2}\left(\left(y_{n_{q_{2}}}^{2}\right)_{t_{2}}\right) & \\ \quad+\sum_{t_{2}<t_{j}<t} T\left(t, t_{j}\right) I_{j}\left(\left(y_{n_{q_{2}}}\right)_{t_{j}}\right) & \\ \quad+\int_{t_{2}}^{t} T(t, s) f_{n_{q_{2}}}(s) d s, & \text { dla } t>t_{2} .\end{cases}
$$

We proceed up to $m$ and find a convergent subsequence $\left(y_{n_{q_{m}, m}}\right)_{q_{m} \in Q_{m}}$ of $\left(y_{n}\right)$, where $Q_{m} \subset Q_{m-1}$. Thus we have

$$
y_{n_{q_{m}, m}}(t):= \begin{cases}y_{n_{q_{m}}}^{1}(t), & \text { dla } t \in\left[0, t_{1}\right] \\ y_{n_{q_{m}}}^{2}(t), & \text { dla } t \in\left(t_{1}, t_{2}\right] \\ \vdots & \\ y_{n_{q_{m}}}^{m}(t), & \text { dla } t \in\left(t_{m-1}, t_{m}\right] .\end{cases}
$$

Let $y$ be a limit of the sequence $\left(y_{n_{q_{m}}, m}\right)_{q_{m} \in Q_{m}}$. Denote the sequence $\left(f_{n_{q_{m}}}\right)_{q_{m} \in Q_{m}}$ by $\left(f_{n_{q_{m}(l)}}\right)_{l \geq 1}$.

From $(F 3)_{m}^{\prime}$, the equality $\beta\left(\left\{f_{n_{q_{m}(l)}}(s)\right\}_{l \geq 1}\right)=0$ and the Dunford-Pettis theorem it follows that, up to subsequence, $f_{n_{q_{m}(l)}} \rightarrow f_{0} \in L^{1}\left(\left[0, t_{m}\right], E\right)$. By the continuity of impulse functions $I_{j}$

$$
y(t)=T(t, 0) x(0)+\sum_{0<t_{j}<t} T\left(t, t_{j}\right) I_{j}\left(y_{t_{j}}\right)+\int_{0}^{t} T(t, s) f_{0}(s) d s .
$$


In the original paper we have showed the way how to prove that $f_{0}(s) \in$ $F\left(s,(y[x])_{s}\right)$ for a.e. $s \in\left[0, t_{m}\right]$. We omit details.

Step 4. As in the original paper we show that $S_{m}=\bigcap_{n=1}^{\infty} \mathrm{cl} S_{m}^{n}$ and $\tilde{\beta}\left(\operatorname{cl} S_{m}^{n}\right) \stackrel{n \rightarrow \infty}{\searrow} 0$, where $\tilde{\beta}$ is the Hausdorff measure of noncompactness in $P C\left(\left[0, t_{m}\right], E\right)[x]$.

Step 5. We show (see the original paper) that the sets $\operatorname{cl} S_{m}^{n}$ are contractible, and finish the proof of the theorem by application of the Hyman and Bothe theorems (see Lemma 5 in [1]).

Theorem 1.1 was applied in the original paper to prove the $R_{\delta}$ structure of solution sets for problems on noncompact intervals. Now Theorem 3.1 can be used in an analogous way to obtain

Theorem 3.2. (Comp. Theorem 3.7 in the original paper) Let $E$ be a Banach space and let hypothesis

$(A)^{\infty}\{A(t)\}_{t \in[0, \infty}$ is a family of linear (not necessarily bounded) operators $(A(t): D(A) \subset E \rightarrow E, t \in[0, \infty), D(A)$ a dense subset of $E$ not depending on $t)$ generating an evolution operator $T: \Delta_{\infty} \rightarrow \mathcal{L}(E)$,

hold, where $\triangle_{\infty}=\left\{(t, s) \in \mathbb{R}_{+} \times \mathbb{R}_{+} \mid 0 \leq s \leq t\right\}$. Suppose that the multivalued map $F:[0, \infty) \times \mathcal{C}([-\tau, 0], E) \rightarrow P_{c p, c v}(E)$ satisfies conditions

$(F 1)^{\infty} F(\cdot, c)$ has a strongly measurable selection for every $c \in \mathcal{C}([-\tau, 0], E)$,

$(F 2)^{\infty} F(t, \cdot)$ is u.s.c. for a.e. $t \geq 0$,

$(F 3)^{\infty} F$ for almost every $t \geq 0$ has at most a linear growth, i.e., there exists a function $\alpha \in L_{\text {loc }}^{1}([0, \infty))$ such that

$$
\|F(t, c)\| \leq \alpha(t)\left(1+\|c\|_{\mathcal{C}}\right) \quad \text { for a.e. } t \in[0, \infty),
$$

$(F 4)^{\prime \infty}$ for every $\varepsilon>0$ and every bounded set $D \subset \mathcal{C}([-\tau, 0], E)$ there exist $\delta>0$ and a function $\mu \in L_{l o c}^{1}([0, \infty))$ such that

$$
\beta\left(F\left(t, O_{\delta}(D)\right)\right) \leq \mu(t) \sup _{-\tau \leq \theta \leq 0} \beta\left(O_{\varepsilon}(D(\theta))\right) \quad \text { for a.e. } t \geq 0 .
$$

Moreover, assume that the maps $I_{k}: \mathcal{C}([-\tau, 0], E) \rightarrow E, k \in \mathbb{N}$, are continuous and there exist constants $r_{k}>0$ such that

$$
\beta\left(I_{k}(D)\right) \leq r_{k} \sup _{-\tau \leq \theta \leq 0} \beta(D(\theta))
$$

for every bounded $D \subset \mathcal{C}([-\tau, 0], E)$.

Then the solution set for problem $(2)$ is an $R_{\delta}$-set in $P C([0, \infty), E)[x]$, where

$$
P C([0, \infty), E)[x]:=\{y[x] \in P C([-\tau, \infty), E) \mid y \in P C([0, \infty), E)\} .
$$

Proof. As in the original paper we observe that $\left\{S_{m}, p_{m}^{k}, \mathbb{N}\right\}$, where $p_{m}^{k}: S_{k} \rightarrow$ $S_{m}, p_{m}^{k}(y)=\left.y\right|_{\left[-\tau, t_{m}\right]}$ for $k \geq m$, forms an inverse system with the limit $S=$ $\lim S_{m}$ equal to the solution set for problem (1). Now, we apply Proposition 3.2 in [2] to conclude that $S$ is also an $R_{\delta}$-set. 


\section{References}

[1] Bothe, D.: Multivalued perturbations of $m$-accretive differential inclusions. Israel J. Math. 108, 109-138 (1998)

[2] Gabor, G.: Acyclicity of solution sets of inclusions in metric spaces. Topol. Methods Nonlinear Anal. 14, 327-343 (1999)

Grzegorz Gabor and Agata Grudzka

Faculty of Mathematics and Computer Science

Nicolaus Copernicus University

Chopina 12/18

87-100 Toruń

Poland

e-mail: ggabor@mat.umk.pl

Agata Grudzka

e-mail: agata33@mat.uni.torun.pl 